\title{
Trabajo social en la gestión del talento humano: de lo operativo a lo estratégico
}

\author{
Melba Yesmit Chaparro Maldonado* \\ Miguel Urra Canales**
}

RESUMEN

Este artículo presenta los resultados producto de la investigación "La acción profesional del Trabajador Social en la Gestión del Talento Humano". Tras entrevistar a 15 profesionales, se identificó la definición de los objetivos, proceso metodológico y funciones del trabajo social en la gestión del talento humano. Los objetivos reflejan un enfoque claramente estratégico, proyectando la labor profesional en el mediano y largo plazo. El proceso metodológico está orientado a la elaboración, implementación y evaluación de un plan de desarrollo del talento humano. Finalmente, las funciones apuntan a aspectos estratégicos de las compañías de los que, en gran medida, depende el éxito empresarial y el desarrollo personal de los trabajadores.

Palabras clave: Trabajo social - Talento humano - Organización

\section{Trabalho social na gestão do talento humano: do operativo ao estratégico}

\section{RESUMEM}

Este artigo apresenta os resultados da investigação "a ação profissional do Trabalhador Social na Gestão do Talento Humano". Trás entrevistar a 15 profissionais, se identificou a definição dos objetivos, processo metodológico e funções do trabalho social na gestão do talento humano. Os objetivos refletem um enfoque claramente estratégico, projetando a labor profissional no mediano e longo prazo. O processo metodológico está orientado a elaboração, implementação e avaliação de um plano de desenvolvimento do talento humano. Finalmente, as funções apontam a aspectos estratégicos das companhias, das que em grande medida, depende o êxito empresarial e o desarrolho pessoal dos trabalhadores.

Palavras chave: Trabalho social - Talento humano - Organização

* Colombiana. Trabajadora Social. Especialista en Administración y Magíster en Docencia, Docente e investigadora del programa de Trabajo Social de la Fundación Universitaria Monserrate (Bogotá) y coordinadora del Semillero de Investigación en Trabajo Social Laboral (SILATS). Correo electrónico: mychaparro@academia.fum.edu.co

** Español. Trabajador Social y Sociólogo. Diplomado en Estudios avanzados dentro del Programa de Doctorado en Ciencias Humanas y Sociales. Docente e investigador del programa de Trabajo Social de la Fundación Universitaria Monserrate (Bogotá). Correo electrónico:murra@academia.fum.edu.co 


\section{Social work in human talent management: from the operation to the strategic}

\section{ABSTRACT}

This article presents the outcomes of the research entitled "The Professional Action of Social Workers in Human Talent Management". Interviews were conducted with 15 professionals, defining objectives, the methodological process and the roles of social work in human talent management. The objectives have a strategic focus and project professional performance into the short and the long-term. The methodological process is targeted at the elaboration, implementation and evaluation of a human talent development plan. The roles of social work are linked to companies' strategic aspects which depend on company success and the personal development of its workers.

Key words: social work - human talent - organisation

\section{Introducción}

La gestión del talento humano, como área, se ha venido posesionando como una aliada estratégica en las organizaciones, capaz de participar en el diseño y ejecución de políticas y estrategias relacionadas con el capital humano, gestando cambios en la cultura organizacional, identificando las potencialidades de los colaboradores como una ventaja competitiva en el desarrollo no solo de la empresa sino de las personas, estableciendo relaciones apropiadas con los diferentes equipos de trabajo, todo lo cual ha significado nuevos desafíos para quienes lideran esta área.

Coherente con lo anterior, el presente artículo tiene como propósito dar a conocer los resultados fruto del proceso de investigación denominado "La acción profesional del Trabajador Social en la Gestión del Talento Humano", desarrollado desde el "Semillero de Investigación en Trabajo Social Laboral - SILATS" de la Fundación Universitaria Monserrate (Bogotá, Colombia), en donde pretende describir los objetivos, proceso metodológico y funciones emergentes del trabajador social en la gestión del talento humano, partiendo de la base que:

"El Trabajador Social ha desempeñado una labor muy importante en las empresas productivas y de prestación de servicios. El talento humano es el activo más grande que poseen las Organizaciones y es precisamente en este campo donde los profesionales de esta disciplina han actuado 
para preservarlo, Grindándole condiciones de calidad de vida personal y laboral" (Sierra, A.L. 2005:131).

En el desarrollo de la investigación, junto a acciones de tipo meramente operativo o administrativo en las que se ha venido desempeñando el trabajador social, en su incursionar en el ámbito empresarial, en donde se identificaron labores como "organizar un taller de pintura", "entrega de dotación", "inscripción a caja de compensación", "celebración de fechas especiales", "entrega de subsidios" o "celebrar el encuentro de pensionados", ha sido posible identificar una tendencia que sitúa al trabajador o trabajadora social como un aliado realmente estratégico de las organizaciones para la gestión del talento humano.

Esto nos lleva a pensar que la ubicación del trabajador social organizacional realizada en la década de los ochenta como subordinado a la dirección de la empresa, a la sección de personal o a los sindicatos (Kisnerman, N. 1984:161) ha evolucionado hacia una concepción más amplia, relacionada con "el pleno desarrollo y proyección del talento humano, en donde se cristalicen la autonomía, creatividad, participación, identidad, pertenencia y reconocimiento como ejes de su accionar (...) [desde] una concepción plenamente humanista, buscando la dignidad humana y el desarrollo integral del ser, donde se logra expresar la equidad, honestidad, profesionalismo y calidad en su ser y en su hacer" (Pardo, L.P. y Arteaga, P. 2001:30).

\section{Metodología}

La investigación se abordó desde un enfoque mixto, articulando lo cuantitativo con lo cualitativo.

Desde la perspectiva cuantitativa, se recolectaron datos utilizando una encuesta con preguntas cerradas tipo cuestionario, las cuales fueron medidas e interpretadas con herramientas estadísticas. Estos datos están relacionados con las dimensiones de competencias intelectuales y laborales, habilidades, actitudes, conocimientos y macrotendencias.

Desde una perspectiva cualitativa, a través de una entrevista estructurada, se abordaron las categorías relacionadas con el presente artículo: los objetivos, proceso metodológico y funciones del Trabajador Social en la Gestión del talento humano, a partir de la experiencia laboral de los entrevistados. En relación con el manejo de los datos cualitativos se procedió a la categorización y elaboración de matrices descriptivas, posteriormente se clasificó 
la información en subcategorías, una vez realizado lo anterior se identificaron las categorías emergentes que se constituyeron en el insumo para el análisis e interpretación de los resultados.

Se construyó una muestra intencional de quince Trabajadores (as) Sociales que lideran el Área de Gestión del Talento Humano en organizaciones o empresas, públicas (un 13\%) y privadas (87\%), que operan en Colombia en los sectores financiero, automotriz, construcción, floricultor, manufacturero, y tienen su sede en la ciudad de Bogotá. El 93\% de los profesionales eran mujeres. El 53\% de la muestra tenía una experiencia de entre 1 y 3 años en el cargo y el $47 \%$ entre 4 y 7 años. La denominación de los cargos era heterogénea, con un $27 \%$ de jefes del área y, en igualdad de porcentaje, con el $20 \%$, coordinadores, directores y otros; finalmente, un $13 \%$ de consultores. El equipo investigador considera que la representatividad de la muestra es suficiente para esbozar la tendencia hacia lo estratégico en la gestión del talento humano que se presenta en este artículo.

\section{Resultados}

\section{Objetivos del trabajo social en la gestión del talento humano}

Con base en la entrevista realizada, es posible inferir cinco objetivos fundamentales en la labor de gestión del talento humano en las organizaciones. Dichos objetivos, destacan por tener un enfoque claramente estratégico, proyectando la labor profesional en el mediano y largo plazo; en lo estructural más que en lo puntual y cortoplacista.

El primero de ellos consistiría en ser un aliado estratégico de la organización que capta talentos y promueve el desarrollo humano y profesional de los colaboradores. Aunque algunas de las respuestas señalaron aspectos operativos como "que las personas que están desarrollando su labor estén bien entrenadas", las principales afirmaciones que sustentan este objetivo son las siguientes: 
Tabla: Objetivos expresados en las entrevistas y análisis de su componente estratégico.

\begin{tabular}{|l|l|}
\hline $\begin{array}{l}\text { Objetivo expresado por el } \\
\text { profesional entrevistado }\end{array}$ & Análisis y reflexión desde el componente estratégico \\
\hline $\begin{array}{l}\text { "Captar talento para nuestras } \\
\text { empresas y clientes" }\end{array}$ & $\begin{array}{l}\text { No se trata sólo de buscar un empleado para } \\
\text { cumplir una función o funciones puntuales, sino } \\
\text { de captar talento; no sólo para la empresa sino } \\
\text { también con proyección hacia el cliente. }\end{array}$ \\
\hline $\begin{array}{l}\text { "Fomentar que el colaborador } \\
\text { cumpla, fortalezca y mejore sus } \\
\text { procesos" }\end{array}$ & $\begin{array}{l}\text { Se va más allá de revisar el cumplimiento de } \\
\text { funciones de los empleados en el día a día, } \\
\text { fortaleciendo y mejorando los procesos con } \\
\text { proyección de futuro. }\end{array}$ \\
\hline $\begin{array}{l}\text { "Contribuir a que las personas } \\
\text { se sientan más felices en sus } \\
\text { trabajos" }\end{array}$ & $\begin{array}{l}\text { El concepto de felicidad en el trabajo incluye } \\
\text { variables, como el salario, incentivos, el clima } \\
\text { laboral o las posibilidades de crecimiento futuro } \\
\text { que ofrece una compañía a sus empleados. }\end{array}$ \\
\hline $\begin{array}{l}\text { "Promover el crecimiento, } \\
\text { aprendizaje y desarrollo, } \\
\text { alineados con los objetivos } \\
\text { personales y corporativos" }\end{array}$ & $\begin{array}{l}\text { La combinación de los objetivos personales y } \\
\text { corporativos hace referencia a un gana-gana entre } \\
\text { empleado y compañía en el mediano y largo plazo. }\end{array}$ \\
\hline
\end{tabular}

Fuente: Elaboración propia.

El segundo objetivo haría referencia a establecer la coherencia y retroalimentación entre los objetivos organizacionales en busca de la satisfacción y logro de los objetivos individuales de los colaboradores. Aunque todavía algunos entrevistados se limitaban al aspecto administrativo, con expresiones como "cumplir con los procesos de rentabilidad de la organización", las respuestas de corte estratégico son contundentes: "Desarrollar programas ajustados a lo que necesita el negocio y lo que necesita el empleado" o "lograr buenos resultados con gente motivada, gente que llegue feliz a trabajar".

El tercer objetivo estratégico estaría orientado a responder a los desafíos de la organización y del contexto social. No se trataría, entonces, de mirar sólo hacia el interior de la organización, sino de tener en cuenta las dinámicas sociales cambiantes y de construir organizaciones abiertas y permeables a sus contextos. Por ejemplo, uno de los profesionales entrevistados señalaba la importancia de "liderar procesos que soporten los retos del negocio" y otro resaltaba la importancia de "formular programas en gestión humana para que la empresa siga siendo competitiva, innovadora y atractiva."

Un cuarto objetivo invitaría a velar por la calidad de vida de los colaboradores y sus familias a través de programas y procesos. 
Resulta especialmente interesante la inclusión de la familia del colaborador, ofreciendo "bienestar laboral para los empleados, a nivel laboral y familiar", así como el hecho de no nombrar actividades o tareas operativas y sí hacer referencia a "procesos de bienestar laboral", "procesos de salud ocupacional" o a "planear, diseñar, ejecutar, coordinar y manejar programas de cultura organizacional, de calidad de vida y de responsabilidad social".

Por último, generar procesos de liderazgo se convertiría en el quinto objetivo estratégico emergente identificado en la investigación, con expresiones como "generar una marca de liderazgo en las personas", "generar estrategias de liderazgo" o "capacitar líderes para que ellos implementen las políticas". La identificación, generación y desarrollo de procesos de liderazgo es uno de los principales ejes estratégicos de las empresas del siglo XXI.

\section{Proceso metodológico del trabajo social en la gestión del talento humano}

Los profesionales que simplemente ejecutan tareas desde un punto de vista operativo no requieren de un proceso metodológico para realizar su labor y cumplir sus objetivos. Sin embargo, un profesional que trabaja desde una perspectiva estratégica otorga gran importancia a la metodología que va a guiar y dotar de sentido el desarrollo de sus acciones. El equipo de investigación, al hacer el análisis de la información recolectada, descubrió en las respuestas de los profesionales entrevistados la posibilidad de establecer un proceso metodológico básico para la gestión del talento humano desde la perspectiva del trabajo social. Este proceso constaría de seis pasos:

a) Elaboración de un diagnóstico y línea base, a través de la recolección de datos, sistematización de experiencias previas y análisis de la información.

b) Establecimiento de perfiles y funciones de los trabajadores de la organización.

c) Detección de necesidades y potencialidades de los trabajadores.

d) Elaboración de un plan de desarrollo del talento humano en la organización, a través del ciclo del PHVA (Planear, hacer, verificar y actuar) y el diseño de planes estratégicos con participación de los ejecutivos de la organización.

e) Socialización del plan con los diferentes directores y jefes de áreas o procesos; esto se logra a través de la estrategia 
del liderazgo, con lo que se identifican las potencialidades de los colaboradores, propiciando de esta manera el desarrollo de la persona en la organización.

f) Evaluación y validación del plan, esta etapa refiere a la identificación de parámetros críticos en el desempeño de los planes y/o programas, en relación con el cumplimiento de los objetivos, su desarrollo y efectividad como consecuencia de su implementación.

Estos seis puntos estarían alineados con los objetivos del plan estratégico de la compañía y con equipos de gestión del talento humano que conciben a las personas como asociadas de la organización, dentro de modelos orgánicos, ágiles, flexibles y cambiantes. Las personas dejarían de ser un problema dentro de las relaciones industriales y pasarían a convertirse en la solución, dejarían de ser mano de obra para convertirse en talentos, seres dotados de inteligencia, capacidades, conocimientos y habilidades (Chiavenato, I. 2009:43).

\section{Funciones del trabajo social en la gestión del talento humano}

De acuerdo a los resultados de la investigación, desde la acción profesional se hace referencia a los retos del Trabajador Social en el área Gestión de Talento Humano, donde aporta al cumplimiento de las metas y a la implementación de estrategias dentro de los lineamientos organizacionales. Respecto a las funciones emergentes centradas en el componente estratégico, éstas se direccionan hacia la formulación de políticas, administración del personal, el mantenimiento y desarrollo de las personas en la organización, el fomento de la participación de los colaboradores y la optimización del bienestar laboral. 
Gráfico: Principales funciones del trabajo social en la gestión del talento humano identificadas en la investigación.

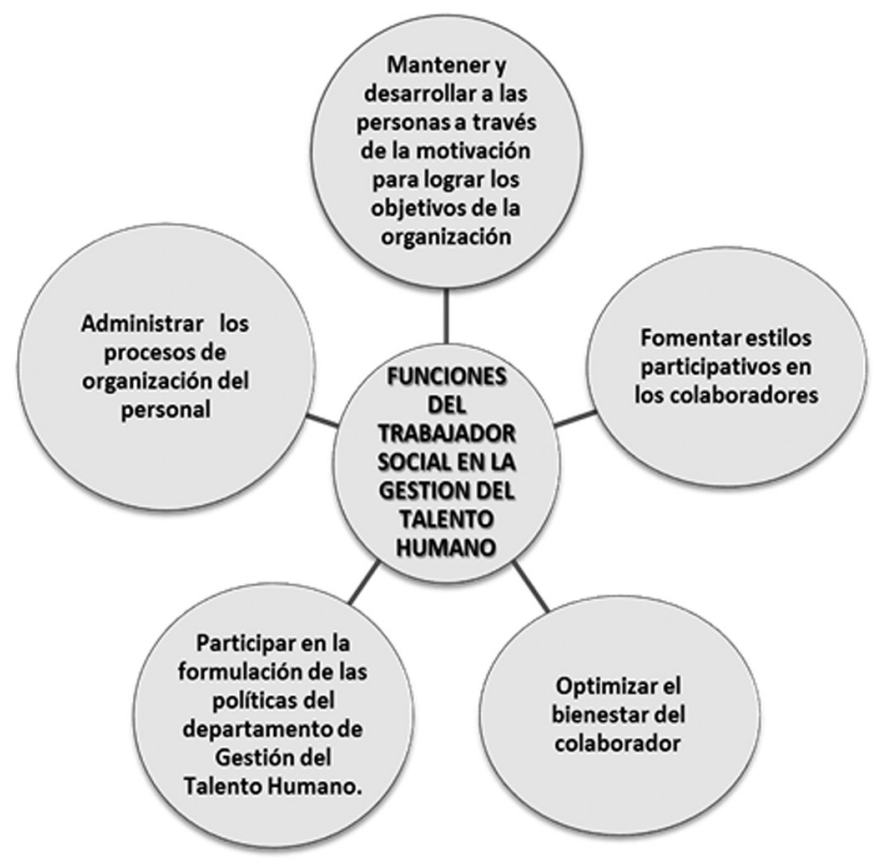

Fuente: elaboración propia.

En cuanto a la participación en la formulación de las políticas del departamento de Gestión del Talento Humano, es importante destacar cómo los cambios en la política de empleo y en la legislación laboral, producto de los procesos de globalización, plantearon nuevas perspectivas para el desempeño del Trabajador Social, en especial aquellas dirigidas a asesorar a nivel gerencial las políticas empresariales enfocadas en los empleados. Esto le ha planteado nuevos retos acerca del impacto en el diseño de estas políticas, tal y como expresan los profesionales entrevistados:

"Participar en el diseño de la estrategia de liderazgo para el desarrollo de los directores $y$ de los gerentes para que sean líderes enfocados en resultados pero que también entiendan que esos resultados los van a lograr a través de la gente."

"Definición de las políticas y estrategias para los procesos de selección y promoción del personal de la organización." 
La perspectiva que han de desarrollar las organizaciones debe estar centrada en las personas, lo que pone de manifiesto el otorgar la importancia y el valor que éstas poseen. En este sentido, los profesionales de trabajo social deben desarrollar una serie de estrategias que promuevan el liderazgo con el propósito ultimo de involucrar a los colaboradores en la visión de los retos organizacionales: "Planteamiento de la estrategia de liderazgo", "La definición e implementación de toda la estrategia de un líder."

Respecto a la administración de los procesos de organización del personal en la empresa, es otro de los aspectos fundamentales en la gestión de talento humano y que puede entenderse como una acción transversal, pues se efectúa a lo largo de toda la vida laboral (Atehortúa, F.A. et al. 2008:159). Por ello, el Trabajador Social, en respuesta al cumplimiento de la normatividad, de la legislación laboral y de las políticas de personal establecidas en las empresas, es responsable de acciones relacionadas con la selección, inducción, contratación, re inducción, certificación de cargos y evaluación de desempeño, tal y como manifestaron los y las profesionales entrevistados:

"Búsqueda y selección de personas para cargos técnicos, profesionales y de alta gerencia."

"Participar en los procesos de pre-ingreso para la selección de personal."

"Identificación de los cargos claves de la compañia."

"Procesos de evaluación."

"Inducción a la organización."

"Elaboración de instrumentos que permiten medir el desempeño de cada una de las personas de la empresa por área."

"Soy responsable del proyecto de certificación por competencias para el personal."

"Apoyo a los procesos de contratación."

"Participar en la re-inducción del personal."

Una tercera función está relacionada con mantener y desarrollar a las personas a través de la motivación para lograr los objetivos de la organización. En los procesos de la gestión del talento humano los colaboradores se reconocen como seres humanos y no como recursos empresariales. Se tienen en cuenta y se respetan sus 
características y diferencias individuales, puesto que están dotados de inteligencia, aptitudes, conocimientos y habilidades específicas. En las organizaciones exitosas ya no se habla de administrar a las personas sino con las personas, considerando a los trabajadores como socios aliados de la empresa (Chiavenato, I. 2003). Bajo las anteriores premisas, los Trabajadores Sociales tienen hoy en día la responsabilidad de promover y estimular el contexto humano de la organización, lo que da un nuevo significado a la dinámica empresarial. Las palabras de los y las profesionales entrevistados parecen estar en línea con Carlos Eduardo Torres, cuando afirma que "el trabajador social organizacional es el profesional que conoce la dinámica de crecimiento y desarrollo de la persona en la organización"(2005:118):

"Desarrollo de potenciales en la organización; entonces básicamente es asegurar que tengamos personas listas para asumir cargos de superintendente, para asumir cargos de supervisores, o para asumir cargos de gerencia."

"Velar porque las personas que están desarrollando sus labores, estén capacitadas, motivadas e incentivadas en donde pueden alinear los sueños que tienen como trabajadores con los sueños de la compañía."

"Soy la persona encargada de dos procesos que denominamos talent management y liderazgo."

"Identifico las capacidades, habilidades y competencias de los colaboradores."

"Promover procesos de desarrollo en el equipo de consultores del área de Gestión del talento Humano."

"Desarrollo de programas de capacitación, retención y monitoreo de personas."

"Motivación a los colaboradores."

Otra de las funciones identificadas en la investigación consiste en fomentar estilos participativos en los colaboradores. La participación de los trabajadores en las organizaciones de hoy encuentra su cimiento en los principios democráticos y de cooperación. Consolidar esta cultura es otra de las funciones que le han sido encomendadas a los Trabajadores Sociales en la línea de asesoría, tanto a la gerencia como a todas las áreas de la empresa, en busca de la satisfacción del colaborador, generando habilidades y motivaciones tanto a nivel individual como colectivo: 
"Ser una promotora de la participación de todos y cada uno de los miembros de los equipos de trabajo en la organización."

"Sistematizo y elaboro el consolidado de lo expresado en el buzón de sugerencias."

"Convoco y lidero los procesos de los comités tanto de salud ocupacional como grupos primarios."

"Ser el interlocutor entre el empleado y la empresa."

La última de las funciones reflejadas en las entrevistas hace referencia a optimizar el bienestar del colaborador. Considerar el bienestar del empleado desde un punto de vista estratégico es crucial para lograr el éxito, la eficiencia y productividad en la empresa. Cada trabajador pasa una parte importante de su vida en la organización, por lo que su mayor bienestar en ella va a redundar en forma determinante en su calidad de vida (Rodríguez, D. 1996:105). Es así que Trabajador Social dentro de esta área ha planteado una serie de propuestas encaminadas al fortalecimiento de la calidad de vida laboral y personal del trabajador, lo que se ha abordado a partir de programas y proyectos de bienestar laboral, capacitaciones técnicas y de desarrollo personal, procesos de orientación familiar y psicosocial o estrategias para optimizar el clima y la comunicación organizacional:

"Soy la profesional responsable del bienestar y clima organizacional."

"Tengo la responsabilidad de la implementación del programa de bienestar, cultura y vida."

"Lidero los proyectos de desarrollo y bienestar para el empleado y su familia."

"Brindo apoyo psicosocial y resolución de conflictos para los colaboradores."

"Mi función consiste en desarrollar el proyecto de bienestar laboral, que incluye actividades de capacitación, recreación y desarrollo humano".

"Acompañamiento a los empleados cuando tienen dificultades que llevan al ausentismo."

En definitiva, las funciones del trabajo social en la gestión del talento humano apuntan a aspectos estratégicos de las compañías de los que, en gran medida, depende el éxito empresarial y el desarrollo personal de los trabajadores. 


\section{Conclusiones}

Esta investigación ha presentado unos resultados que indican la tendencia del trabajador social a asumir un rol estratégico en la gestión del talento humano de las organizaciones.

Esta tendencia se concreta en la definición por parte de los profesionales entrevistados de los objetivos estratégicos, el proceso metodológico y las funciones del trabajo social en la gestión del talento humano, lo que plantea retos profesionales en un contexto en constante evolución y cambio. Todo esto, desde actitudes de objetividad y creatividad, visión holística de la organización, trabajo en equipo e interdisciplinar, disposición al cambio, liderazgo y lealtad aliados con los principios del trabajo social.

Los objetivos reflejan un enfoque claramente estratégico, proyectando la labor profesional en el mediano y largo plazo: promoción del desarrollo humano de los colaboradores, integración de los objetivos individuales con los objetivos organizacionales, responder a los retos de la organización y del contexto sin afectar la parte humana de la empresa y generar procesos de liderazgo. En el proceso metodológico, se mantienen las etapas propias de la planeación en trabajo social, enfocadas en el desarrollo del talento humano. Para finalizar, las funciones presentan una tendencia a la proactividad en los procesos, llamando al Trabajador Social a no ser un mero ejecutor de tareas, sino a participar, proponer, administrar, formular políticas y aportar una visión de corte más gerencial en la gestión del talento humano.

Los Trabajadores y Trabajadoras Sociales que desempeñan su labor profesional en la gestión del talento humano afrontan el reto de mantener la consolidación de su presencia en la esfera estratégica de las organizaciones, desde donde pueden promover el cambio organizacional orientado por los principios de ética, equidad, redistribución, responsabilidad social, solidaridad y compromiso con las personas; abriendo una tercera vía en el dilema existente entre la parte humana y la parte productiva de las empresas.

Este escenario abre nuevas posibilidades para la definición de las organizaciones como campo de acción profesional para el trabajo social y nuevas líneas de investigación sobre las competencias laborales del trabajador social en la gestión del talento humano 
de acuerdo a este nuevo perfil estratégico identificado, que plantearían a su vez nuevas preguntas de investigación: ¿Cuáles serían las diferencias entre los nuevos roles del trabajador social en las organizaciones y los que desempeñaba anteriormente? ¿Qué nuevas metodologías utiliza el trabajador social en el contexto empresarial? ¿Qué diferencia al trabajador social organizacional de otras profesiones que también abordan la gestión del talento humano?

\section{Bibliografía}

Atehortúa, F.A.; Bustamante, R.E. y Valencia, J.A. (2008). Sistema de gestión integral. Una sola gestión, un solo equipo. Medellín: Universidad de Antioquia.

Chiavenato, I. (2003). Gestión del Talento Humano. Bogotá: McGraw-Hill. Chiavenato, I. (2009). Gestión del talento humano. México: McGraw-Hill.

Kisnerman, N. (1984). Introducción al Trabajo Social. Buenos Aires: Humanitas.

Pardo, L. P. y Arteaga, P. (2001). Gestión social del talento humano. Buenos Aires: Lumen Humanitas.

Rodríguez, D. (1996). Gestión organizacional. Elementos para su estudio. México: Plaza y Valdéz.

Sierra, A. L. (2005). El Trabajo Social en las organizaciones: Haciendo historia. Revista de la facultad de Trabajo Social. Universidad Pontificia Bolivariana, 21, 131-135.

Torres, C.E. (2005). Trabajo social en organizaciones: Nueva alternativa y perspectiva profesional. Revista Colombiana de Trabajo Social, 19. 